\title{
Entanglement wedge minimum cross-section in holographic massive gravity theory
}

\author{
Peng Liu, Chao Niu, Zi-Jian Shi and Cheng-Yong Zhang ${ }^{1}$ \\ Department of Physics and Siyuan Laboratory, Jinan University, \\ Guangzhou 510632, China \\ E-mail: phylp@email.jnu.edu.cn, niuchaophy@gmail.com, \\ szj@stu2016.jnu.edu.cn, zhangcy@email.jnu.edu.cn
}

AbSTRACT: We study the entanglement wedge cross-section (EWCS) in holographic massive gravity theory, in which a first and second-order phase transition can occur. We find that the mixed state entanglement measures, the EWCS and mutual information (MI) can characterize the phase transitions. The EWCS and MI show exactly the opposite behavior in the critical region, which suggests that the EWCS captures distinct degrees of freedom from that of the MI. More importantly, EWCS, MI and HEE all show the same scaling behavior in the critical region. We give an analytical understanding of this phenomenon. By comparing the quantum information behavior in the thermodynamic phase transition of holographic superconductors, we analyze the relationship and difference between them and provide two mechanisms of quantum information scaling behavior in the thermodynamic phase transition.

KEYwORDS: AdS-CFT Correspondence, Black Holes, Holography and condensed matter physics (AdS/CMT)

ArXIV EPRINT: 2104.08070

${ }^{1}$ Corresponding author. 


\section{Contents}

1 Introduction 1

2 Holographic massive gravity theory 2

3 The holographic entanglement entropy 5

4 The holographic mutual information $\quad 9$

5 The entanglement wedge minimum cross-section 11

6 The critical behavior of the geometry-related quantities 14

$\begin{array}{lll}7 & \text { Discussion } & 16\end{array}$

\section{Introduction}

As a special property of the quantum system, quantum information plays an important role in other fields, such as condensed matter theory and holographic gravity theory. Many quantum phase transitions involving strong correlations could be characterized by entanglement measures [1-4]. Recently, holographic gravity theory has built a bridge between quantum information and geometry and has sparkled important insights into the geometric correspondence of quantum information properties and the understanding of the emergence of spacetime [5-8].

There are many different measures of quantum entanglement, such as entanglement entropy (EE) and mutual information (MI), which characterize different properties of quantum systems. It is worth noting that EE, which is the most concerned physical quantity, is not suitable for describing the entanglement of mixed states, while the concepts of MI, Rényi entropy, entanglement of purification, reflected entropy and entanglement negativity are more suitable to describe the entanglement of mixed states [9, 10]. A longstanding problem with quantum entanglement is that it is usually extremely difficult to calculate.

In recent years, holographic duality theory has been widely used to study strongly correlated physics, and it bridges the gap between geometry and quantum information. The earliest development is that the entanglement entropy of quantum field theory is proportional to the area of the minimum surface in dual gravity theory, which has been dubbed as the holographic entanglement entropy (HEE) [5]. HEE has been proved a good diagnose of quantum phase transitions and thermodynamic phase transitions [1123]. Subsequently, many other holographic duals of quantum information related physical quantities have been proposed, and their applications in strong correlation theory have been studied [11-15]. For example, Rényi entropy has been proposed to be proportional 
to the area of the minimum cosmic brane [24]. Entanglement of purification, reflected entropy, odd entropy and entanglement negativity have been proposed to be proportional to the area of the entanglement wedge minimum cross-section (EWCS) [25-30]. The EWCS provides a novel and powerful tool for studying the mixed state entanglement [31-51]. In addition, quantum complexity has been associated with the volume or action of certain region. Moreover, the butterfly velocity, a dynamical quantum information property of the quantum system, has been related to the geometry of the horizon of the black hole [37, 5260]. All these developments pave the way for the study of quantum information properties of strongly correlated systems in the framework of holographic duality theory.

Massive gravity plays an important role in the holographic duality theory because it can break the translational symmetry, thus producing momentum dissipation in the dual condensed matter system [61-67]. As an important theory of gravity, its entanglement property has been systematically studied [68]. However, the mixed state entanglement property in massive gravity theory has not been well studied. Therefore, the main goal of this paper is to study the mixed state entanglement in massive gravity - the EWCS. In particular, we will discuss the properties of EWCS and its comparison with HEE and MI. It is noted that there is a thermodynamic phase transition (Hawking-Page transition) in massive gravity theory [61,68-70]. Remind also that in the holographic superconductivity phase transition model, the EWCS has been found to have obvious non-smoothness at the critical points, thus diagnosing the thermal phase transitions [71]. Therefore, it is desirable to examine what role the mixed entanglement measures play during the phase transitions in massive gravity theory. More importantly, whether the quantum entanglement during superconductivity phase transition is different from that of Hawking-Page phase transition, and the underlying reasons are worth studying.

We organize this paper as follows: we introduce the AdS massive gravity model in section 2. We discuss the properties of HEE (3), MI (4) and EWCS (5) systematically. In 6, we explore the scaling behavior of EWCS, MI and HEE. Finally, we summarize in section 7 .

\section{Holographic massive gravity theory}

The action of $n+2$-dimensional massive gravity system reads [69],

$$
S=\frac{1}{16 \pi G_{n}} \int d^{n+2} x \sqrt{-g}\left[R+\frac{n(n+1)}{L^{2}}-\frac{1}{4} F^{2}+m^{2} \sum_{i}^{4} c_{i} \mathcal{U}_{i}(g, f)\right],
$$

where $G_{n}$ is the n-dimensional Newton constant which we set as $1, f_{\mu \nu}$ is the reference metric, $c_{i}$ are constants and $\mathcal{U}_{i}$ are symmetric polynomials of the eigenvalue of the $(n+$ 2) $\times(n+2)$ matrix $\mathcal{K}^{\mu}{ }_{\nu} \equiv \sqrt{g^{\mu \alpha} f_{\alpha \nu}}$,

$$
\begin{aligned}
& \mathcal{U}_{1}=[\mathcal{K}], \\
& \mathcal{U}_{2}=[\mathcal{K}]^{2}-\left[\mathcal{K}^{2}\right], \\
& \mathcal{U}_{3}=[\mathcal{K}]^{3}-3[\mathcal{K}]\left[\mathcal{K}^{2}\right]+2\left[\mathcal{K}^{3}\right], \\
& \mathcal{U}_{4}=[\mathcal{K}]^{4}-6\left[\mathcal{K}^{2}\right][\mathcal{K}]^{2}+8\left[\mathcal{K}^{3}\right][\mathcal{K}]+3\left[\mathcal{K}^{2}\right]^{2}-6\left[\mathcal{K}^{4}\right] .
\end{aligned}
$$


Here [.] denotes the trace. The $F^{2} \equiv F^{\mu \nu} F_{\mu \nu}$ is the square of the Maxwell field strength. $L$ is the AdS length scale, which we fix as 1 for convenience. $m$ introduces mass to the graviton, which breaks the translational symmetry. The translational symmetry will be recovered when $m \rightarrow 0$.

The action (2.1) admits a solution [69],

$$
d s^{2}=-f(r) d t^{2}+f^{-1}(r) d r^{2}+r^{2} h_{i j} d x^{i} d x^{j}, \quad i, j=1,2,3, \cdots, n .
$$

with the reference metric

$$
f_{\mu \nu}=\operatorname{diag}\left(0,0, c_{0}^{2} h_{i j}\right)
$$

The Maxwell field reads,

$$
A_{t}=\mu-\frac{Q}{(n-1) r^{n-1}}
$$

where the chemical potential $\mu$ can be obtained by requiring a vanishing static electric potential,

$$
\mu=\frac{Q}{(n-1) r_{+}^{n-1}}
$$

The $\mathcal{U}_{\#}$ are

$$
\begin{aligned}
& \mathcal{U}_{1}=n c_{0} / r \\
& \mathcal{U}_{2}=n(n-1) c_{0}^{2} / r^{2}, \\
& \mathcal{U}_{3}=n(n-1)(n-2) c_{0}^{3} / r^{3}, \\
& \mathcal{U}_{4}=n(n-1)(n-2)(n-3) c_{0}^{4} / r^{4},
\end{aligned}
$$

and the function $f$ is

$$
\begin{aligned}
f(r)= & k+\frac{r^{2}}{L^{2}}-\frac{M}{r^{n-1}}+\frac{Q^{2}}{2 n(n-1) r^{2(n-1)}}+\frac{c_{0} c_{1} m^{2}}{n} r+c_{0}^{2} c_{2} m^{2} \\
& +\frac{(n-1) c_{0}^{3} c_{3} m^{2}}{r}+\frac{(n-1)(n-2) c_{0}^{4} c_{4} m^{2}}{r^{2}} .
\end{aligned}
$$

$M, Q$ are the mass and the charge of the black hole. $h_{i j} d x^{i} d x^{j}$ is the line element of the Einstein space whose curvature is $n(n-1) k$. Therefore, we can denote the case of spherical, Ricci flat and hyperbolic horizon as $k=1,0,-1$. We consider the 4-dimensional flat case $(k=0)$ in this paper, where $\mathcal{U}_{3}=\mathcal{U}_{4}=0$. Consequently, the last two terms in (2.8) vanishes. Note also that $m$ and $c_{i}$ in (2.8) are redundant, we denote $\alpha \equiv c_{0} c_{1} m^{2} / 2, \beta \equiv$ $c_{0}^{2} c_{2} m^{2}$ such that

$$
f(r)=-\frac{2 M}{r}+\frac{Q^{2}}{4 r^{2}}+r^{2}+\alpha r+\beta .
$$

At the horizon $r=r_{h}$ we have $f\left(r_{h}\right)=0$. We can solve $M$ with $r_{h}$ and the Hawking temperature reads

$$
T=\frac{f^{\prime}\left(r_{h}\right)}{4 \pi}=-\frac{Q^{2}-4 r_{h}^{2}\left(\beta+2 \alpha r_{h}+3 r_{h}^{2}\right)}{16 \pi r_{h}^{3}} .
$$




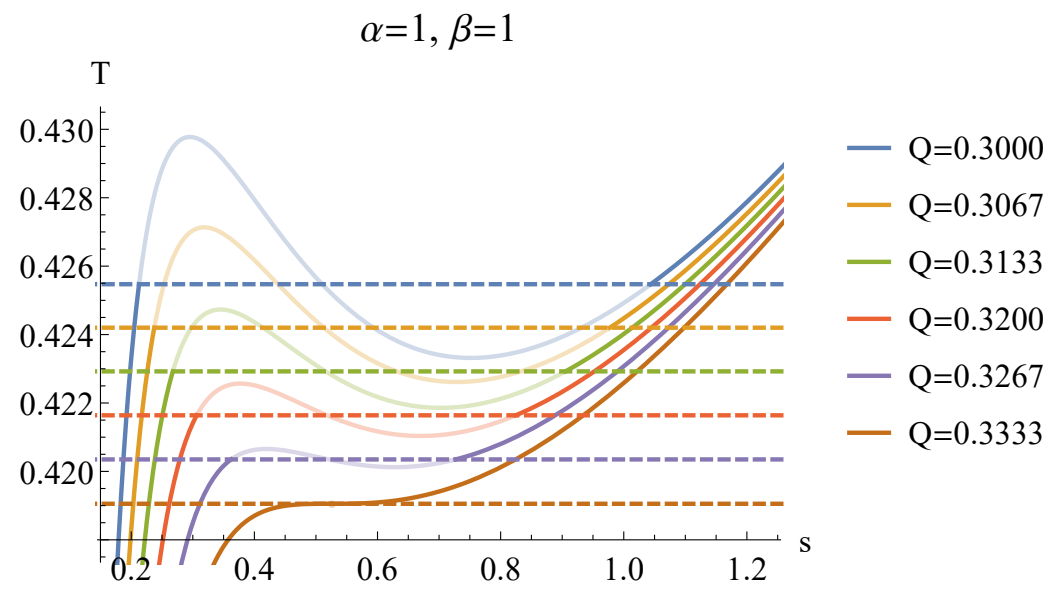

Figure 1. The phase transition at several different values of the $Q$. The solid lines are the $T-s$ relation at $Q$ specified by the plot legends, where the lighter segments correspond to the metastable regions. The dashed horizontal lines, of which the parameters match the color of the plot legends, are the critical temperature where the phase transition occurs. The brown curve $Q=0.3333$ corresponds to the critical case of the second-order phase transition.

The effective entropy density ${ }^{1}$ is $s \equiv \pi r_{h}^{2}$. We can rewrite the temperature as

$$
T=\frac{-\pi^{2} Q^{2}+8 \sqrt{\pi} \alpha s^{3 / 2}+12 s^{2}+4 \pi \beta s}{16 \pi^{3 / 2} s^{3 / 2}} .
$$

Solving $\partial_{s} T=0$ we have two roots,

$$
s=\frac{\pi}{6}\left(\beta \pm \sqrt{\beta^{2}-9 Q^{2}}\right) .
$$

From the above equation, we can find that the van der Waals phase transition occurs when the above two roots are all positive, i.e.,

$$
\beta \geqslant 3 Q
$$

We are particularly interested in the phase transition process. The first-order phase transition $(\beta>3 Q)$ occurs when the entropy density jumps. Meanwhile, the second-order phase transition $(\beta=3 Q)$ occurs when the entropy density is continuous while its first derivative to temperature is discontinuous.

We show an example of $(\alpha, \beta)=(1,1)$ in figure 1 , from which we can find that the system undergoes a van der Waals like thermal phase transition for $Q<1 / 3$. At $Q=$ $1 / 3$, the system undergoes a second-order phase transition. Further increasing the $Q$, the

\footnotetext{
${ }^{1}$ For $k=0$ case, the entropy diverges due to the infinitely large plane, therefore a density is adopted to discuss this problem. The entropy density, defined by dividing the entropy by the area is supposed to be $r_{h}^{2} / 4$. However, to facilitate the discussion of thermodynamics, it is more convenient to define the effective entropy density such that $F=M-T s$ can effectively capture the phase transitions. See [69] for a more complete formalism.
} 
thermal phase transition is absent. Near the critical point $\beta=3 Q$ where second-order phase transition occurs, as can be seen from figure 1 , there is

$$
s^{\prime}(T) \rightarrow \infty
$$

This suggests that a critical scaling behavior will emerge in the critical region. The entropy density and temperature at the critical point are respectively

$$
s_{c}=\frac{\pi Q}{2}, \quad T_{c}=\frac{\alpha+2 \sqrt{2 Q}}{2 \pi} .
$$

Near the critical point, there is

$$
\left(s-s_{c}\right) \sim\left(T-T_{c}\right)^{\alpha_{s}},
$$

in which the exponent $\alpha_{s}$ is called the critical exponent of the entropy density. Given the temperature expression, one can obtain

$$
s^{\prime}(T)=\frac{32 \pi^{3 / 2} s^{5 / 2}}{3 \pi^{2} Q^{2}+12 s^{2}-4 \pi \beta s} .
$$

Expanding (2.17) near $s=s_{c}$ as

$$
s^{\prime}(T)=\frac{\sqrt{2} \pi^{4} Q^{5 / 2}}{3\left(s-\frac{\pi Q}{2}\right)^{2}}+\frac{5 \sqrt{2} \pi^{3} Q^{3 / 2}}{3\left(s-\frac{\pi Q}{2}\right)}+\frac{5 \pi^{2} \sqrt{Q}}{\sqrt{2}}+O\left(\left(s-\frac{\pi Q}{2}\right)^{1}\right),
$$

we find that the leading order is $s^{\prime}(T) \sim\left(s-s_{c}\right)^{-2}$. Together with (2.16), one can immediately obtain the critical exponent as

$$
\alpha_{s}=1 / 3 .
$$

Similar analysis can also be found in [68].

\section{The holographic entanglement entropy}

Now we study the entanglement structure of the system. Entanglement, as an important feature that distinguishes quantum systems from classical systems, can be described by many physical quantities. One of the most famous is the EE. For a system composed of $A$ and $B$, the properties of $A$ are described by a reduced density matrix $\rho_{A}=\operatorname{Tr}_{B} \rho_{\text {total }}$ where $\rho_{\text {total }}$ is the density matrix of the whole system. EE is defined as the von Newmann entropy of the reduced density matrix $\rho_{A}$,

$$
S_{A}(|\psi\rangle)=-\operatorname{Tr}\left[\rho_{A} \log \rho_{A}\right] .
$$

For a system in pure state $|\psi\rangle, \rho_{A}=\operatorname{Tr}_{B}(|\psi\rangle\langle\psi|)$. The above definition leads to $S_{A}=$ $S_{B}$ [72]. EE has been widely recognized as a good entanglement measure for pure states. However, it is not suitable for describing the entanglement of mixed states. The reason is that the degrees of freedom in $A$ and $B$ in the direct product state $\rho_{A} \otimes \rho_{B}$ are not 

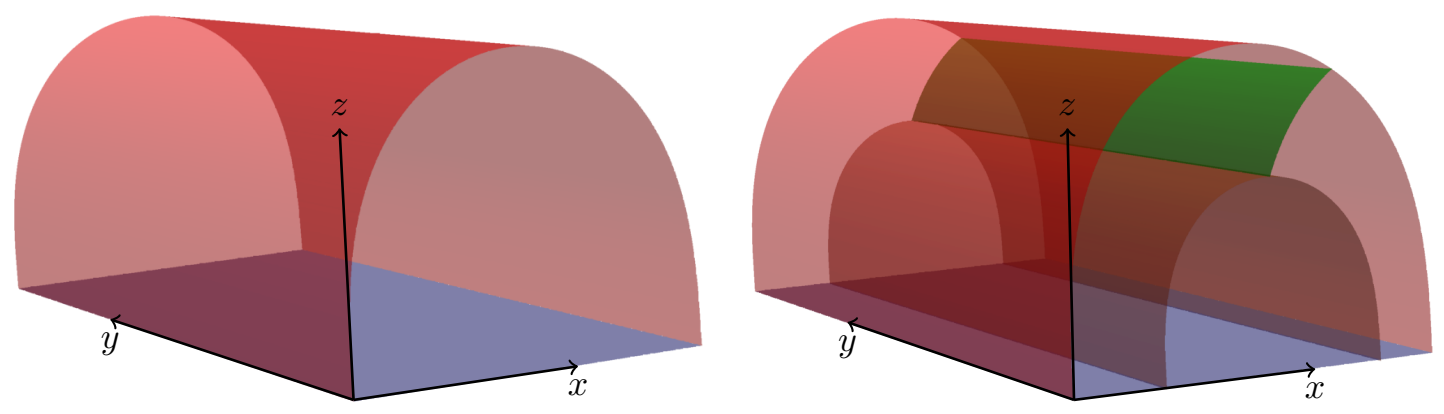

Figure 2. The left plot: the minimum surface for a given width $w$. The right plot: the minimum cross-section (green surface) of the entanglement wedge.

entangled, but they can have non-zero EE. Several new entanglement measures have been proposed to characterize the entanglement of mixed states, among which MI is the most commonly used one $[9,10]$.

In holographic duality theory, the $\mathrm{EE}$ is related to the minimum area stretching into the bulk of the dual gravity systems [5] (see figure 2). Here, we consider the infinite strip along $y$-direction, thus the minimum surfaces will be invariant along $y$-direction. Adopting the angle as the parameterization, the minimum surface can be solved efficiently (see figure 11). In this method, the range of angle is $(0, \pi / 2)$. The first step of our numerical method is to discretize the angle with Gauss-Lobatoo collocation [73]. Because the equations of motion for the minimal surface are nonlinear, we need to apply Newton-Raphson iteration method to find the minimum surface based on the discretization.

First, we discuss the HEE during the first-order phase transition where $(Q, \alpha, \beta)=$ $(0.2,1.0,1.0)$. We find that the relationship between the HEE and temperature depends on the configuration, which is completely different from other black holes, such as AdS-RN black holes [16]. Figure 3 is the HEE at small configurations, from which we can see that the HEE decreases with increasing temperature. The segments with lighter colors correspond to the metastable regions. Moreover, by comparing the HEE at different widths (different curves) in this figure we can find that the HEE increases with the increase of the width $w$. The black dotted line in the figure shows the critical temperature $T_{c}=0.4439$ of the first-order phase transition. When the temperature drops to the critical temperature, HEE will jump abruptly.

When crossing the critical point of the first-order phase transition, as shown in figure 2 , the horizon radius $r_{h}$ of the black hole will jump abruptly. Therefore, the sudden jump of geometry produces the sharp jump of the HEE.

When the configuration size increases, HEE gradually presents a more complicated phenomenon. It can be seen from figure 4 that with the decrease of temperature, HEE decreases first. But when the temperature drops below the critical temperature, the HEE increases with the decrease of temperature. Next, we give an understanding of these phenomena. Firstly, the decrease of HEE with the decrease of temperature in a large configuration can be understood as the contribution of thermodynamic entropy. In figure 1 , 


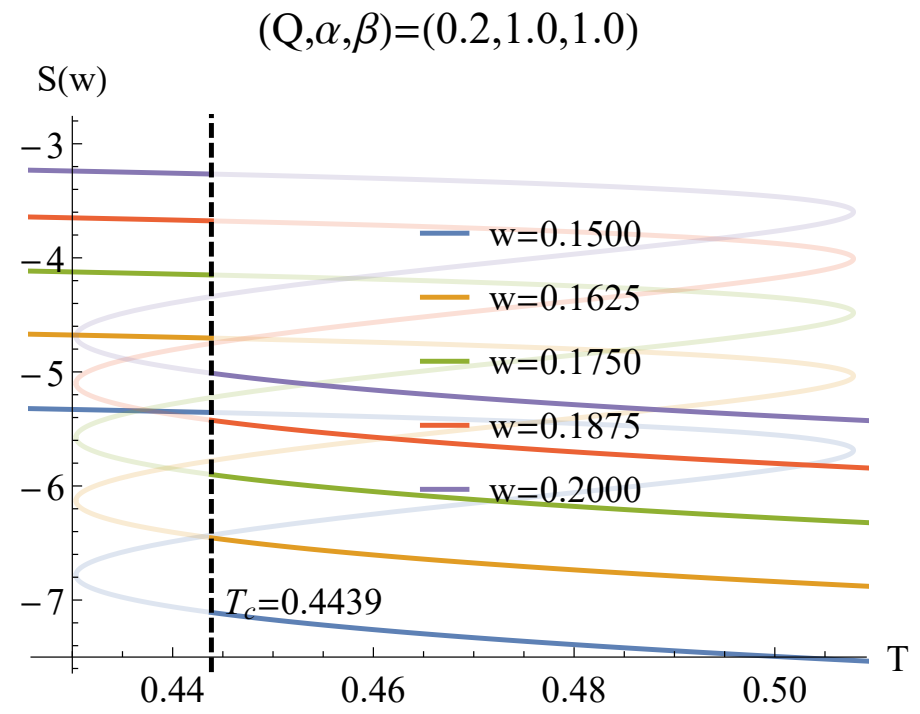

Figure 3. HEE vs. $T$ at several small widths, where the lighter segments correspond to the metastable regions. The red dashed horizontal line represents the critical temperature $T_{c}=0.4439$ of the first-order phase transition.

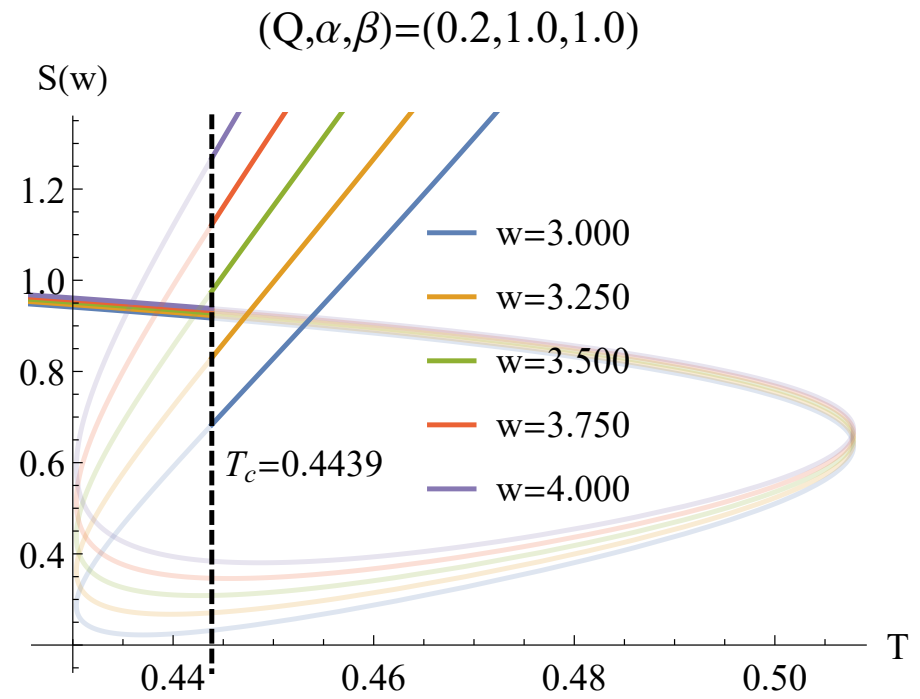

Figure 4. HEE vs. $T$ at several large widths specified by the plot legends, where the lighter segments correspond to the metastable regions. The black dashed vertical line represents the critical temperature $T_{c}=0.4439$ of the first-order phase transition. For each solid curve, the segments with lighter color are the unstable regions.

the entropy density decreases rapidly with the decrease of temperature above the critical temperature. Therefore, the entanglement entropy decreases rapidly above the critical temperature. However, when the temperature drops below the critical temperature, it can be seen from figure 1 that the entropy density changes very slowly with temperature. The behavior of entropy density with temperature no longer dominates the change of HEE 

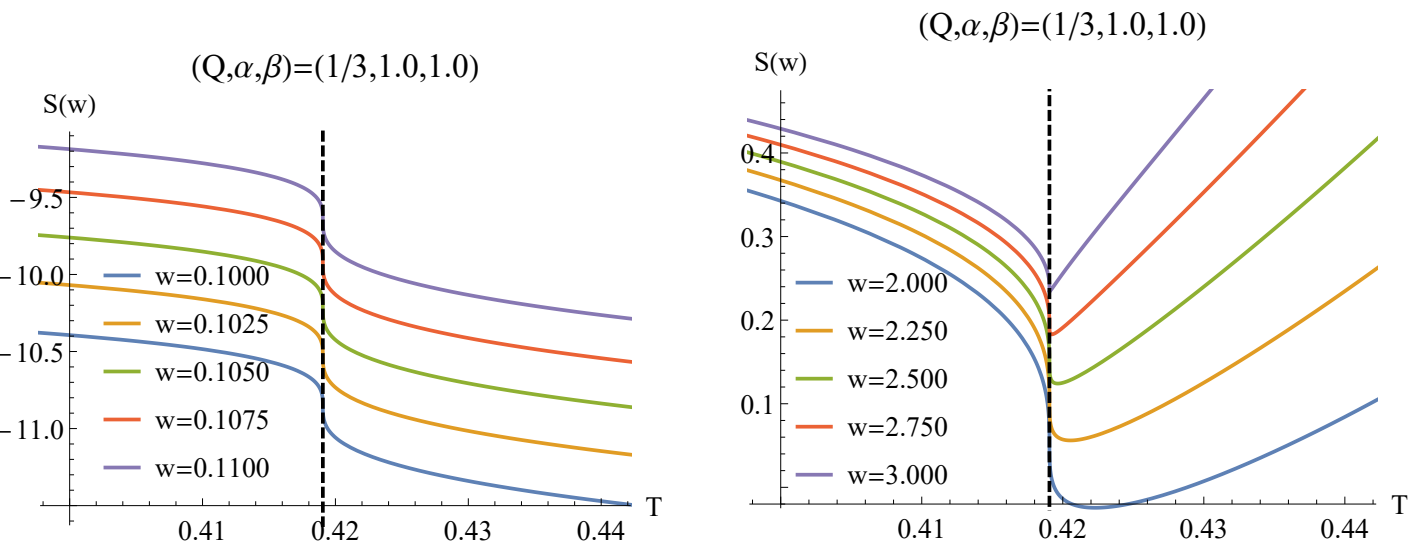

Figure 5. The relationship between the temperature and the HEE for small (left plot) and large widths (right plot) at the critical case with second-order phase transition. The red dashed horizontal line represents the critical temperature $T_{c}=0.4191$ of the second-order phase transition.

with temperature, instead, the whole bulk geometry controls the change of HEE with temperature.

Now, we study the second-order phase transition with $(Q, \alpha, \beta)=(1 / 3,1,1)$. Regardless of the configuration, HEE exhibits a singular behavior at the critical temperature (see figure 5). This is the response of HEE for the second-order phase transition. The HEE behavior at the second-order phase transition point is still related to the specific configuration. When the configuration is small (left figure in figure 5), HEE increases with the decrease of temperature. However, when the configuration is large (right figure in figure 5), HEE first decreases with the decrease of temperature, but increases after passing the critical point. The dependence of HEE on configuration size before and after the second-order phase transition can also be understood by comparing the contribution of thermodynamic entropy with that of whole bulk geometry (see the similar discussion in the previous paragraph).

Finally, we discuss the case without phase transition with $(Q, \alpha, \beta)=(0.5,1,1)$. From figure 6 we find that, when the configuration is small, HEE increases with the decrease of temperature. However, when the configuration is large, the HEE decreases first and then increases with the decrease of temperature. These phenomena are quite different from other black holes, such as AdS-RN black holes.

The above phenomena indicate that the HEE behavior in the massive gravity model can diagnose the first and second-order phase transitions, and the behavior of the HEE is closely related to specific configurations. Only when the temperature is relatively high or the configuration is relatively large, the HEE will increase with the temperature. This is as expected, because a higher temperature or larger subregion will render the minimum surface closer to the event horizon of the black hole, and its HEE will be dominated by thermodynamic entropy.

After elaborating on the properties of HEE, we then discuss the properties of MI, one of the entanglement measures of mixed states. 

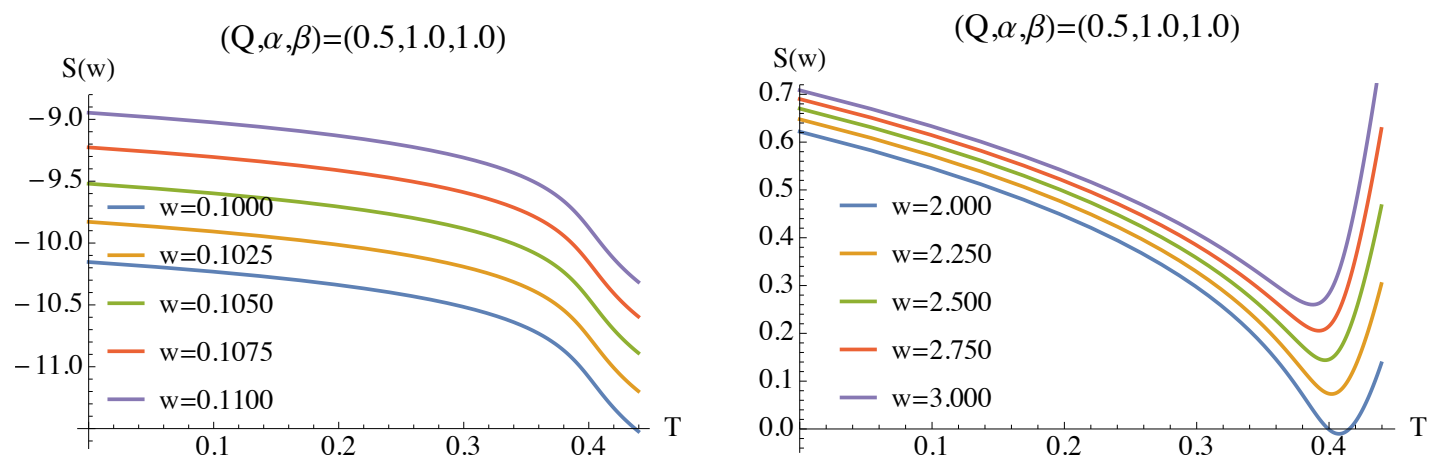

Figure 6. The relationship between the temperature and the HEE for small (left plot) and large widths (right plot) in absence of thermal phase transitions.

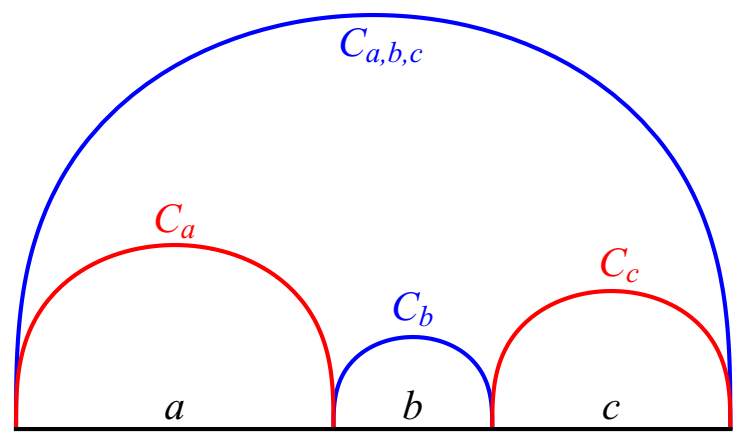

Figure 7. The demonstration of mutual information.

\section{The holographic mutual information}

For disjoint subregion $A \cup C$ with separation $B$, the MI is defined as

$$
I(A, C):=S(A)+S(C)-S(A \cup C),
$$

which can measure the entanglement between $A$ and $C$. It is then straightforward to verify that $I(A, C)=0$ when $\rho_{A C}=\rho_{A} \otimes \rho_{C}$. Therefore, MI can recognize that the direct product state has no entanglement. Because the definition of MI is closely related to HEE, we can directly study the properties of MI based on HEE calculation. The minimum surface corresponding to $S(A \cup C)$ has two candidates, the red curves $C_{a} \cup C_{c}$ and blue curves $C_{b} \cup C_{a, b, c}$ in figure 7 , in which we have labeled the width of $A, B, C$ with $a, b, c$, respectively.

First, we focus on the first-order phase transition at $(Q, \alpha, \beta)=(0.2,1.0,1.0)$. As can be seen from figure 8 , when the configuration is small, the behavior of MI with temperature is similar to that of HEE (see figure 3). This behavior can be derived from the definition of the relationship between MI and HEE. Since the HEE of small configuration changes more rapidly with temperature (as can be seen by comparing HEE at the small and large configuration in figure 6), the behavior of MI with temperature is dominated by the part with the minimum width. Therefore, $\mathrm{MI}$ in a small configuration is qualitatively consistent 


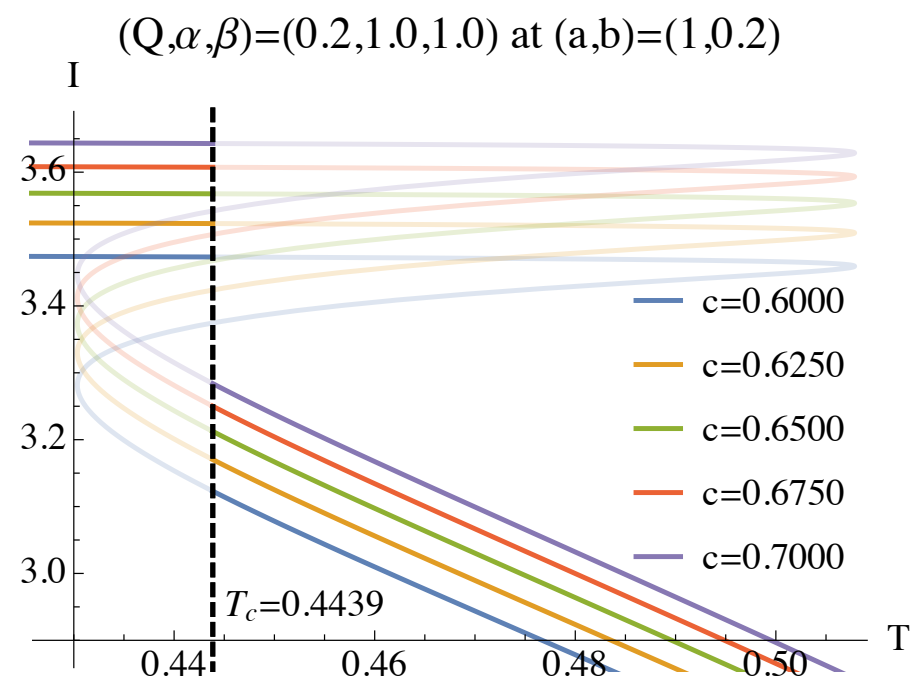

Figure 8. The $T$ vs. $I$ in the scenario with a first-order phase transition, where the lighter segments correspond to the metastable regions. The red dashed horizontal line represents the critical temperature $T_{c}=0.4439$ of the first-order phase transition.

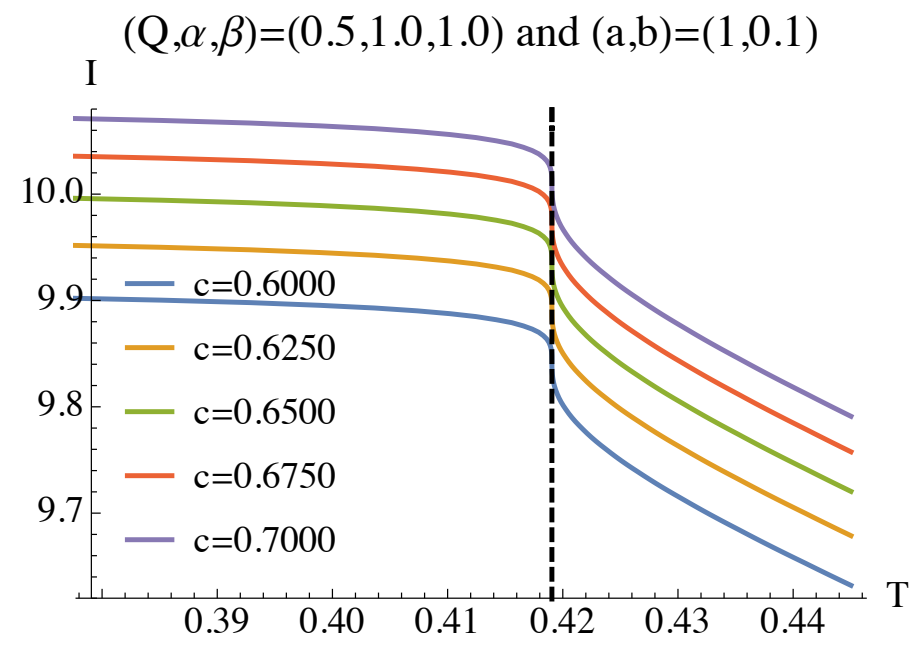

Figure 9. The $T$ vs. $I$ in the scenario with a second-order phase transition. The red dashed horizontal line represents the critical temperature $T_{c}=0.4191$ of the second-order phase transition.

with that of HEE. It is noteworthy that, unlike the apparent configuration-dependent behavior of HEE, these phenomena of MI are configuration-independent.

Now, we study the behavior of MI when the second-order phase transition occurs. First, similar to the monotonic behavior at the first-order phase transition, MI again increases with decreasing temperature (see figure 9). Moreover, the behavior of MI becomes singular at the critical temperature of the second-order phase transition. This is the reflection of the second-order phase transition. In addition, by comparing the MI at different c's (different curves), we can see that MI always increases with the increase of $c$. This phenomenon is in line with expectations because larger configurations mean more entanglement between separate subregions. 


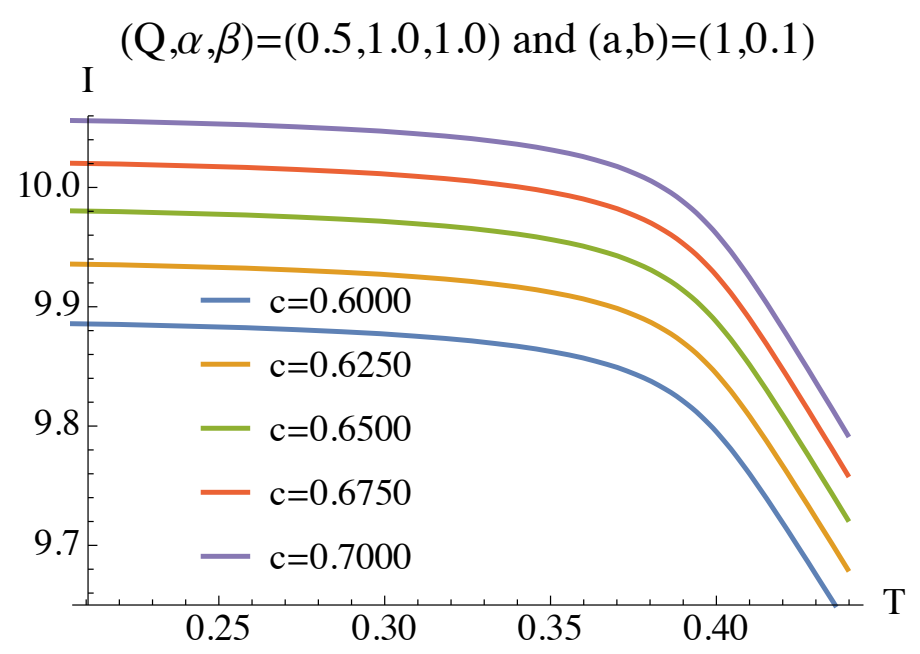

Figure 10. The $T$ vs. $I$ in the case without thermal phase transitions.

Finally, we study the behavior of MI in the absence of phase transition. As can be seen from figure 10, that MI increases monotonically with decreasing temperature. This phenomenon is consistent with the cases in presence of phase transitions.

We summarize the behavior of MI in massive gravity. Firstly, MI can reflect the first-order and second-order phase transitions of massive gravity, with discontinuous and singular behavior at the critical temperatures, respectively. Secondly, the MI increases with decreasing temperature, independent of the specific configuration or system parameter. From the above phenomena, we can see that MI is directly determined by HEE in certain cases. Therefore, MI is not a perfect measure of entanglement of mixed States. We need to resort to other mixed-state entanglement measures. Next, we study the EWCS, a novel mixed state entanglement measure.

\section{The entanglement wedge minimum cross-section}

Recently, several entanglement measures related to the purification process have attracted extensive attention, such as entanglement of purification, reflected entropy, and so on [2729]. These measures of mixed state entanglement have been proposed dual to EWCS, which indicates that EWCS is a widely accepted geometric dual of entanglement of mixed states. Takayanagi proposed that the entanglement of purification $E_{W}\left(\rho_{A B}\right)$ is proportional to the area of the EWCS $\Sigma_{A B}[25]$,

$$
E_{W}\left(\rho_{A B}\right)=\min _{\Sigma_{A B}}\left(\frac{\operatorname{Area}\left(\Sigma_{A B}\right)}{4 G_{N}}\right) .
$$

When the MI is zero, the entanglement wedge becomes disconnected, and hence EWCS vanishes because the cross-section does not exist.

Solving EWCS is difficult for three-fold reasons. First, the equations of motion for solving the minimal surface are highly nonlinear. Second, the minimum cross-section, which minimizes the cross-section with each cross-section itself a local minimum surface, 
is usually hard to find. Last but not least, in the presence of the black hole, the numerical precision is easily sabotaged by the coordinate singularity near the black hole horizon.

Recently, we proposed an efficient algorithm for solving EWCS by using the property that the minimum cross-section must be locally perpendicular to the boundaries of the entanglement wedge. Figure 11 is a schematic diagram of the main ideas of the algorithm for solving EWCS. Considering the EWCS of two parallel infinite strips along $y$-direction in a homogeneous background,

$$
d s^{2}=g_{t t} d t^{2}+g_{z z} d z^{2}+g_{x x} d x^{2}+g_{y y} d y^{2},
$$

where $z=0$ represents the asymptotic AdS boundary. Due to the translational invariance along $y$-direction, the EWCS will also be invariant along $y$-direction. The homogeneity implies that metric components $g_{\mu \nu}$ are functions of $z$ only. For a biparty subsystem with minimum surfaces $C_{1}\left(\theta_{1}\right), C_{2}\left(\theta_{2}\right)$, we work out the minimum surface $C_{p_{1}, p_{2}}$ connecting $p_{1} \in C_{1}$ and $p_{2} \in C_{2}$. We can parametrize $C_{p_{1}, p_{2}}$ with $z$, and hence the area of $C_{p_{1}, p_{2}}$ reads,

$$
A=\int_{C_{p_{1}, p_{2}}} \sqrt{g_{x x} g_{y y} x^{\prime}(z)^{2}+g_{z z} g_{y y}} d z .
$$

The equation of motion reads,

$$
x^{\prime}(z)^{3}\left(\frac{g_{x x} g_{y y}^{\prime}}{2 g_{y y} g_{z z}}+\frac{g_{x x}^{\prime}}{2 g_{z z}}\right)+x^{\prime}(z)\left(\frac{g_{x x}^{\prime}}{g_{x x}}+\frac{g_{y y}^{\prime}}{2 g_{y y}}-\frac{g_{z z}^{\prime}}{2 g_{z z}}\right)+x^{\prime \prime}(z)=0,
$$

with boundary conditions,

$$
x\left(z\left(\theta_{i}\right)\right)=x\left(\theta_{i}\right), \quad i=1,2 .
$$

The local perpendicular condition between the minimum cross-section and the entanglement wedge implies that

$$
\left\langle\frac{\partial}{\partial z}, \frac{\partial}{\partial \theta_{1}}\right\rangle_{p_{1}}=0, \quad\left\langle\frac{\partial}{\partial z}, \frac{\partial}{\partial \theta_{2}}\right\rangle_{p_{2}}=0
$$

where $\langle\cdot, \cdot\rangle$ represents the vector product measured by the metric $g_{a b}$. For better numerical stability control, we adopt the normalized local orthogonal relation,

$$
\left.Q_{1}\left(\theta_{1}, \theta_{2}\right) \equiv \frac{\left\langle\frac{\partial}{\partial z}, \frac{\partial}{\partial \theta_{1}}\right\rangle}{\sqrt{\left\langle\frac{\partial}{\partial z}, \frac{\partial}{\partial z}\right\rangle\left\langle\frac{\partial}{\partial \theta_{1}}, \frac{\partial}{\partial \theta_{1}}\right\rangle}}\right|_{p_{1}}=0,\left.\quad Q_{2}\left(\theta_{1}, \theta_{2}\right) \equiv \frac{\left\langle\frac{\partial}{\partial z}, \frac{\partial}{\partial \theta_{1}}\right\rangle}{\sqrt{\left\langle\frac{\partial}{\partial z}, \frac{\partial}{\partial z}\right\rangle\left\langle\frac{\partial}{\partial \theta_{2}}, \frac{\partial}{\partial \theta_{2}}\right\rangle}}\right|_{p_{2}}=0 .
$$

Finding the EWCS is turned to locating the minimum surface anchoring at $\left(\theta_{1}, \theta_{2}\right)$ where (5.7) is satisfied. To this end, we adopt the Newton-Raphson method to locate the endpoints satisfying the local perpendicular conditions. ${ }^{2}$ Based on the above techniques, we now study the relationship between the thermal phase transition and the EWCS.

\footnotetext{
${ }^{2}$ It is worth noting that there is a possibility of local minimums for the minimum section. However, our Newton iteration method does capture the global minimum. First, we have tried many different initial guesses for the iteration, all of which converge to the same minimum cross-section. This means that the minimum cross-section will not be densely distributed. Second, because the inner curve $C_{1}$ is close to the AdS boundary, $p_{1}$ can only be distributed near the top of $C_{1}$. Because the minimum cross-section can not be densely distributed, there is no local minimum in the calculation of the minimum cross-section. To sum up, what our algorithm finds is the global minimum indeed.
} 


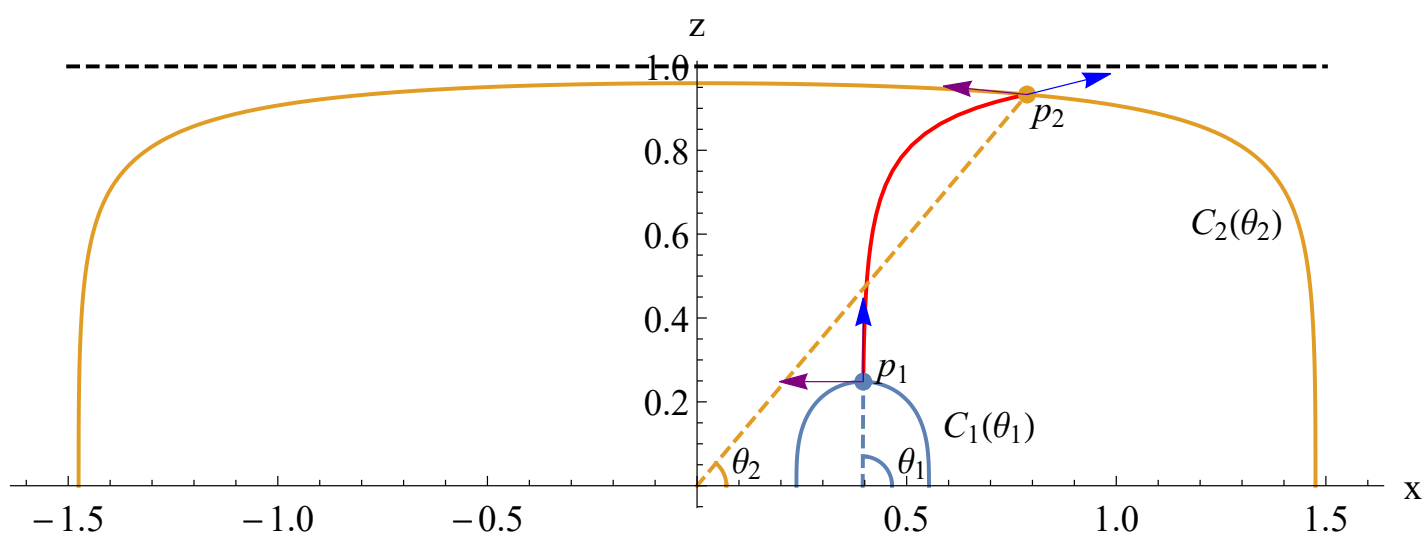

Figure 11. The schematic demonstration of the EWCS. The $p_{1}$ and $p_{2}$ are the intersection of the minimum surface connecting those two minimum surfaces. The solid blue curve (parametrized by $\theta_{1}$ ) and solid orange curve (parametrized by $\theta_{2}$ ) are minimum surfaces. The thick purple curve is the minimum surface connecting $p_{1}$ and $p_{2}$. The blue arrows at the $p_{1}$ and $p_{2}$ are the tangent vectors $\left.\left(\frac{\partial}{\partial z}\right)^{a}\right|_{p_{1}}$ and $\left.\left(\frac{\partial}{\partial z}\right)^{a}\right|_{p_{2}}$ along the $C_{p_{1}, p_{2}}$, while the purple arrows are the tangent vectors $\left.\left(\frac{\partial}{\partial \theta_{1}}\right)^{a}\right|_{p_{1}}$ and $\left.\left(\frac{\partial}{\partial \theta_{2}}\right)^{a}\right|_{p_{2}}$ along $C_{1}, C_{2}$, respectively. The dark dashed horizontal line represents the black brane horizon.

We show the relationship between the EWCS and temperature during the first-order phase transition in figure 12. It can be seen from the figure that the first-order phase transition does have an impact on EWCS. The EWCS also exhibits a van der Waals-like behavior. As the temperature decreases, the EWCS decreases and jumps at the critical temperature of the first-order phase transition. Note that MI increases with decreasing temperature (figure 8), and the EWCS exhibits the opposite behavior. These phenomena of EWCS are also independent of configurations.

Now, we focus on the second-order phase transition. It can be seen from figure 13 that, similar to MI, the EWCS also exhibits a singular behavior at the phase transition. The difference is that here again the behavior of EWCS with temperature is opposite to that of MI. EWCS decreases with decreasing temperature, while MI increases with decreasing temperature (figure 9).

At last, we study the case where no thermal phase transition occurs at $Q>b / 3$. As we can see from figure 14, the EWCS again shows the opposite behavior from the MI (figure 10) for relatively large temperature. For small temperatures, however, we can see that the EWCS starts to show similar behavior as that of the MI.

We summarize the behavior of EWCS in the massive gravity theory. First of all, EWCS can indeed reflect the first-order and second-order phase transitions of the system, which are represented by the jump and singular behavior at the critical temperatures, respectively. In addition, EWCS exhibits an opposite temperature dependence to MI. This shows that EWCS captures distinct degrees of freedom from that of MI. The behavior of MI can be understood by its association with HEE, whereas EWCS cannot. MI can still be dominated by the behavior of HEE in many cases, while EWCS is not controlled by HEE. 


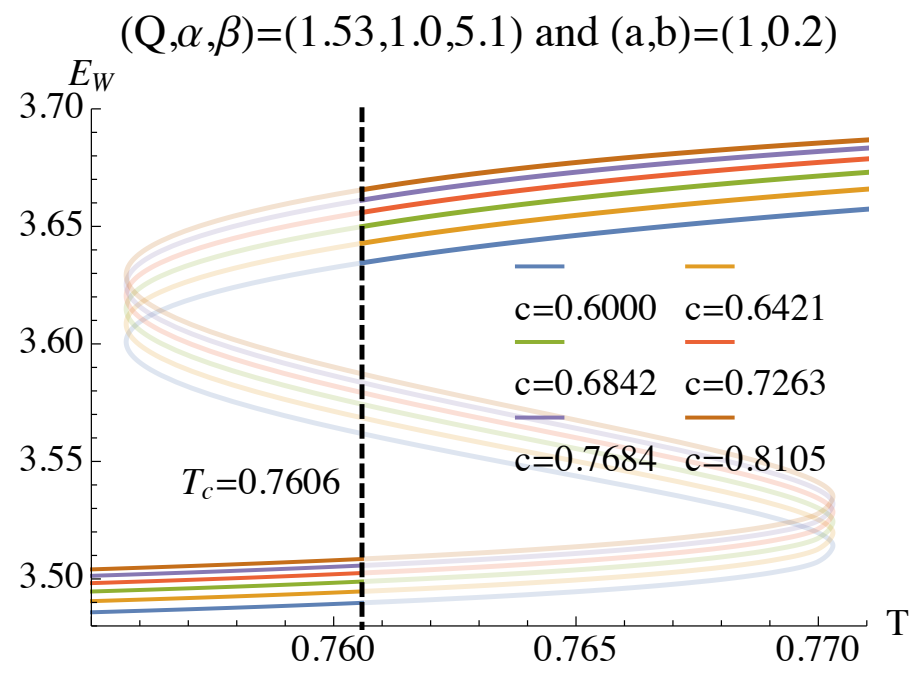

Figure 12. The $T$ vs. EWCS in presence of the first-order phase transition, where the lighter segments correspond to the metastable regions. The red dashed horizontal line represents the critical temperature $T_{c}=0.7547$ of the first-order phase transition.

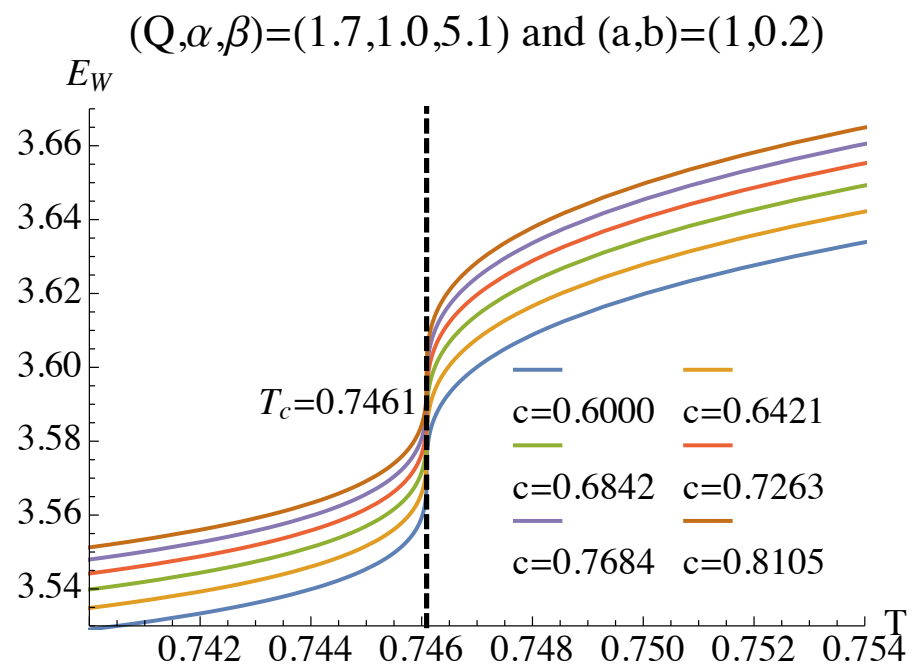

Figure 13. The temperature vs. EWCS at the second-order phase transition. The phenomenon is qualitatively the same for other configurations and parameters. The red dashed horizontal line represents the critical temperature $T_{c}=0.7461$ of the second-order phase transition.

\section{The critical behavior of the geometry-related quantities}

From the above studies on HEE, MI and EWCS, it can be found that these geometry-related entanglement quantities have singular behaviors near the critical point of the second-order phase transition. In fact, we can analytically prove that these three quantities, even any geometrically related physical quantity, will exhibit the same scaling behavior. Moreover, we will also verify the correctness of the analytical analysis with numerical results. 


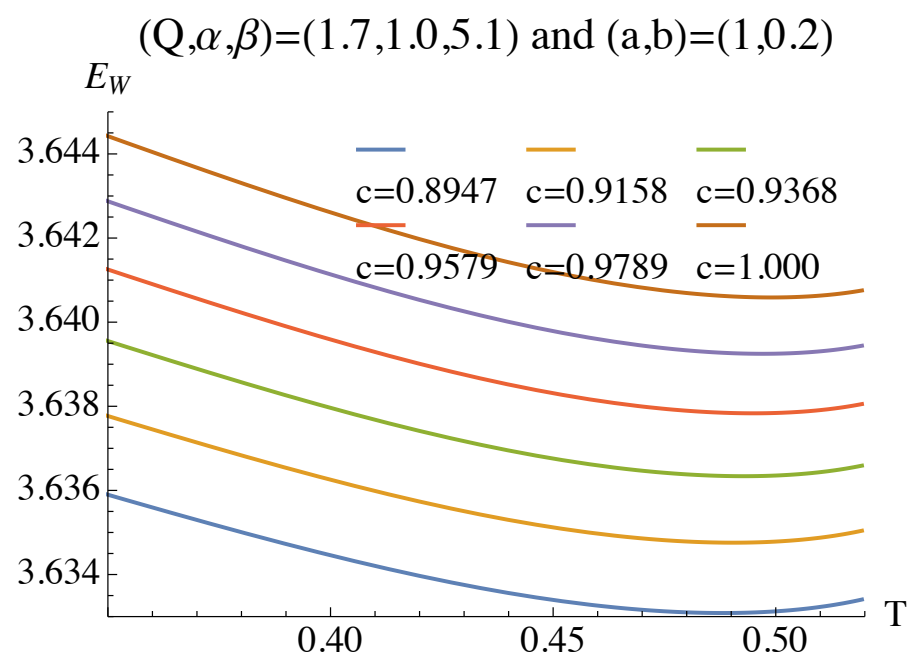

Figure 14. The temperature vs. EWCS at the case where no thermal phase transition occurs.

At the onset of the second-order phase transition, any geometrically related physical quantity $A$ can be expressed as

$$
A=A_{c}+A^{\prime} \delta g_{\mu \nu}
$$

where $A_{c}$ is the value at the critical point. Given that the entropy density behavior (2.19), we will find that,

$$
\delta s \sim \delta g_{\mu \nu} \sim\left(T-T_{c}\right)^{1 / 3} .
$$

Therefore, any geometry-related physical quantities will scale as,

$$
\left(A-A_{c}\right) \sim\left(T-T_{c}\right)^{1 / 3} .
$$

We support this analysis numerically. Figure 15 shows the scaling behavior of HEE with $T$ and EWCS with $T$ during the second-order phase transition. It can be seen from the figure that the logarithm values of $\delta S \equiv S-S_{c}$ and $\delta E_{W} \equiv E_{W}-E_{W_{c}}$ depends linearly on the logarithm values of temperature difference $\delta T \equiv T-T_{c}$, and the slopes converge to $1 / 3$. This means that our analytical analysis and numerical analysis are mutually confirmed.

It is worth mentioning that in another second-order thermodynamic phase transition - holographic superconductor model, we also found a critical behavior of the geometrically related quantities [71]. Although the scaling behavior exists in both the superconductivity phase transition and the Hawking-Page transition, the underlying mechanisms are totally different. The core of all the critical behaviors in this paper comes from the (6.1), that is, the relationship between the change of metric $\delta g_{\mu \nu}$ near the critical point of the second-order Hawking-Page transition. and the change of temperature $\delta T$. Notice that the HawkingPage transition is not a traditional thermodynamic phase transition. For example, there is no spontaneous symmetry breaking and spontaneous condensation of order parameters. However, the holographic superconducting phase transition process is consistent with the traditional phase transition theory. When crossing the critical point, the $\mathrm{U}(1)$ symmetry of the system is spontaneously broken, accompanied by the spontaneous condensation of 

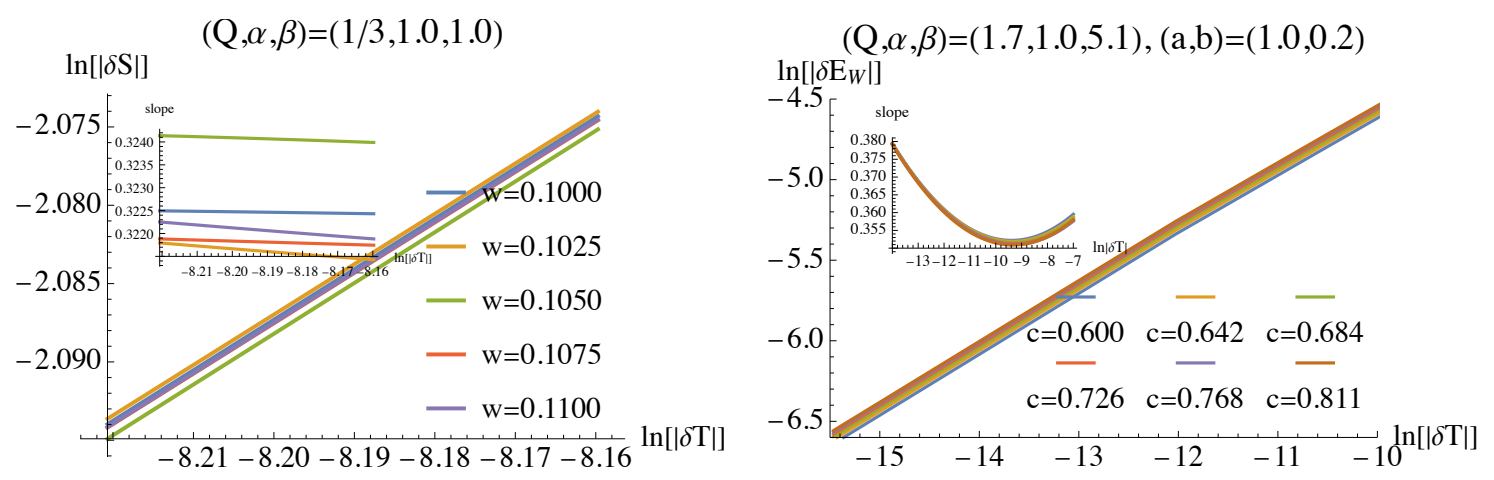

Figure 15. The scaling behavior between HEE and $T$, and EWCS and $T$. The inset in each plot is the slope $\frac{d \ln |\delta \#|}{d \ln |\delta T|}$ vs. $\ln |\delta T|$.

a complex scalar field. It is the appearance of spontaneous condensation that modifies the background geometry. The back-reaction of the background geometry is the square of condensation correction [74], which leads to the critical exponent as $\alpha=1$. While for the thermodynamic phase transition in this paper, all geometry-related physical quantities have a critical exponent of $\alpha=1 / 3$.

\section{Discussion}

In this paper, we studied the mixed state entanglement, as well as the entanglement entropy in the van der Waals phase transition of massive gravity. We find MI, EWCS, as well as HEE, can all characterize the phase transitions. Also, the patterns of characterizing the phase transition for HEE, MI and EWCS are essentially the same: they encounter a jump at the first-order phase transition point and exhibit a singular behavior at the secondorder phase transition point. Moreover, HEE shows obvious configuration dependence. The HEE behavior with temperature is completely different in small configuration and large configuration. As a comparison, the temperature behaviors of MI and EWCS are independent of the configuration. However, we also observe an intriguing phenomenon that MI behavior with temperature is completely the opposite of that of the EWCS in the critical region. Specifically, MI increases with decreasing temperature, while EWCS decreases with decreasing temperature. This phenomenon indicates that there is an important difference between EWCS and MI, especially in the critical regions. Moreover, we can see that MI and HEE are closely related in some cases, which means that EWCS might be a better candidate for the entanglement of mixed states. It is also proved that all geometryrelated physical quantities have the same critical exponent $\alpha=1 / 3$ near the second-order phase transition point, both analytically and numerically. Moreover, this phenomenon is completely different in the phenomenon and in the underlying mechanism from that of superconductivity phase transition. The key reason is that the scaling relationships between the perturbation of the metric (which affects the geometric quantities related to quantum information) and the temperature are different. The superconductivity phase transition is accompanied by the appearance of condensation, but the Hawking-Page phase 
transition is not. This renders them have different scaling behaviors. This conclusion and related techniques can be applied to any physical quantity that only depends on geometric correlation.

In addition to the thermal phase transition accompanied by the emergence of order parameters [75] and the van der Waals-like phase transition, quantum phase transition may exhibit a completely different scenario. Quantum phase transition happens at absolute zero temperature when varying system parameters. In certain kinds of quantum phase transitions involving strong correlation, the order parameter does not emerge. It is then desirable to explore the characterization of these quantum phase transitions. In holographic duality theory, the quantum phase transition is related to the flow among different IR fixed points. However, solving a domain wall with IR fixed points is technically difficult since the zero-temperature limit may be accompanied by singular behaviors. Therefore, we are dealing with the quantum phase transitions at a low whereas nonvanishing temperature. Notice that the HEE, as well as MI in certain limits, can be determined by the thermal entropy, the HEE and MI are not good candidates for characterizing the quantum phase transitions. The EWCS, however, is by definition free of the control from the near horizon behavior. We can expect that the EWCS can work as a good diagnose of the quantum phase transitions. More specifically, we should also expect a scaling law during quantum phase transitions. From the perspective of holography, the quantum phase transition is triggered by the flow between different IR fixed points, which will lead to the abrupt change of geometry. Similar to the scaling law in this paper, we can also expect the scaling law of quantum-information-related physical quantities during quantum phase transition.

\section{Acknowledgments}

Peng Liu would like to thank Yun-Ha Zha for her kind encouragement during this work. This work is supported by the Natural Science Foundation of China under Grant No. 11805083, 11905083, 12005077 and Guangdong Basic and Applied Basic Research Foundation (2021A1515012374).

Open Access. This article is distributed under the terms of the Creative Commons Attribution License (CC-BY 4.0), which permits any use, distribution and reproduction in any medium, provided the original author(s) and source are credited.

\section{References}

[1] A. Osterloh, L. Amico, G. Falci and R. Fazio, Scaling of entanglement close to a quantum phase transitions, Nature 416 (2002) 608 [quant-ph/0202029].

[2] L. Amico, R. Fazio, A. Osterloh and V. Vedral, Entanglement in many-body systems, Rev. Mod. Phys. 80 (2008) 517 [quant-ph/0703044] [InSPIRE].

[3] M. Levin and X.G. Wen, Detecting topological order in a ground state wave function, Phys. Rev. Lett. 96 (2006) 110405 [cond-mat/0510613].

[4] A. Kitaev and J. Preskill, Topological entanglement entropy, Phys. Rev. Lett. 96 (2006) 110404 [hep-th/0510092]. 
[5] S. Ryu and T. Takayanagi, Holographic derivation of entanglement entropy from AdS/CFT, Phys. Rev. Lett. 96 (2006) 181602 [hep-th/0603001] [INSPIRE].

[6] V.E. Hubeny, M. Rangamani and T. Takayanagi, A covariant holographic entanglement entropy proposal, JHEP 07 (2007) 062 [arXiv:0705.0016] [INSPIRE].

[7] A. Lewkowycz and J. Maldacena, Generalized gravitational entropy, JHEP 08 (2013) 090 [arXiv: 1304.4926] [INSPIRE].

[8] X. Dong, A. Lewkowycz and M. Rangamani, Deriving covariant holographic entanglement, JHEP 11 (2016) 028 [arXiv:1607.07506] [INSPIRE].

[9] G. Vidal and R.F. Werner, A computable measure of entanglement, Phys. Rev. A 65 (2002) 032314 [quant-ph/0102117].

[10] R. Horodecki, P. Horodecki, M. Horodecki and K. Horodecki, Quantum entanglement, Rev. Mod. Phys. 81 (2009) 865.

[11] T. Nishioka and T. Takayanagi, AdS bubbles, entropy and closed string tachyons, JHEP 01 (2007) 090 [hep-th/0611035] [INSPIRE].

[12] I.R. Klebanov, D. Kutasov and A. Murugan, Entanglement as a probe of confinement, Nucl. Phys. B 796 (2008) 274 [arXiv: 0709.2140] [inSPIRE].

[13] A. Pakman and A. Parnachev, Topological entanglement entropy and holography, JHEP 07 (2008) 097 [arXiv: 0805.1891] [INSPIRE].

[14] S.-J. Zhang, Holographic entanglement entropy close to crossover/phase transition in strongly coupled systems, Nucl. Phys. B 916 (2017) 304 [arXiv: 1608.03072] [INSPIRE].

[15] X.-X. Zeng and L.-F. Li, Holographic phase transition probed by nonlocal observables, Adv. High Energy Phys. 2016 (2016) 6153435 [arXiv: 1609.06535] [INSPIRE].

[16] Y. Ling, P. Liu, C. Niu, J.-P. Wu and Z.-Y. Xian, Holographic entanglement entropy close to quantum phase transitions, JHEP 04 (2016) 114 [arXiv:1502.03661] [INSPIRE].

[17] Y. Ling, P. Liu and J.-P. Wu, Characterization of quantum phase transition using holographic entanglement entropy, Phys. Rev. D 93 (2016) 126004 [arXiv: 1604.04857] [INSPIRE].

[18] Y. Ling, P. Liu, J.-P. Wu and Z. Zhou, Holographic metal-insulator transition in higher derivative gravity, Phys. Lett. B 766 (2017) 41 [arXiv:1606.07866] [InSPIRE].

[19] X.-M. Kuang, E. Papantonopoulos and B. Wang, Entanglement entropy as a probe of the proximity effect in holographic superconductors, JHEP 05 (2014) 130 [arXiv:1401.5720] [INSPIRE].

[20] H. Guo, X.-M. Kuang and B. Wang, Holographic entanglement entropy and complexity in Stückelberg superconductor, Phys. Lett. B 797 (2019) 134879 [arXiv:1902.07945] [INSPIRE].

[21] S. Mahapatra, Interplay between the holographic QCD phase diagram and mutual \& n-partite information, JHEP 04 (2019) 137 [arXiv: 1903.05927] [INSPIRE].

$[22]$ D. Dudal and S. Mahapatra, Interplay between the holographic QCD phase diagram and entanglement entropy, JHEP 07 (2018) 120 [arXiv: 1805.02938] [INSPIRE].

[23] A. Dey, S. Mahapatra and T. Sarkar, Thermodynamics and entanglement entropy with Weyl corrections, Phys. Rev. D 94 (2016) 026006 [arXiv:1512.07117] [INSPIRE].

[24] X. Dong, The gravity dual of Renyi entropy, Nature Commun. 7 (2016) 12472 [arXiv: 1601.06788] [INSPIRE]. 
[25] T. Takayanagi and K. Umemoto, Entanglement of purification through holographic duality, Nature Phys. 14 (2018) 573 [arXiv:1708.09393] [INSPIRE].

[26] P. Nguyen, T. Devakul, M.G. Halbasch, M.P. Zaletel and B. Swingle, Entanglement of purification: from spin chains to holography, JHEP 01 (2018) 098 [arXiv:1709. 07424] [INSPIRE].

[27] J. Kudler-Flam and S. Ryu, Entanglement negativity and minimal entanglement wedge cross sections in holographic theories, Phys. Rev. D 99 (2019) 106014 [arXiv:1808.00446] [INSPIRE].

[28] Y. Kusuki, J. Kudler-Flam and S. Ryu, Derivation of holographic negativity in $A d S_{3} / C F T_{2}$, Phys. Rev. Lett. 123 (2019) 131603 [arXiv:1907.07824] [INSPIRE].

[29] S. Dutta and T. Faulkner, A canonical purification for the entanglement wedge cross-section, JHEP 03 (2021) 178 [arXiv: 1905.00577] [INSPIRE].

[30] K. Tamaoka, Entanglement wedge cross section from the dual density matrix, Phys. Rev. Lett. 122 (2019) 141601 [arXiv: 1809.09109] [INSPIRE].

[31] R.-Q. Yang, C.-Y. Zhang and W.-M. Li, Holographic entanglement of purification for thermofield double states and thermal quench, JHEP 01 (2019) 114 [arXiv:1810.00420] [INSPIRE].

[32] M. Ghodrati, X.-M. Kuang, B. Wang, C.-Y. Zhang and Y.-T. Zhou, The connection between holographic entanglement and complexity of purification, JHEP 09 (2019) 009 [arXiv: 1902.02475] [INSPIRE].

[33] Y.-f. Huang, Z.-j. Shi, C. Niu, C.-y. Zhang and P. Liu, Mixed state entanglement for holographic axion model, Eur. Phys. J. C 80 (2020) 426 [arXiv:1911.10977] [INSPIRE].

[34] G. Fu, P. Liu, H. Gong, X.-M. Kuang and J.-P. Wu, Holographic informational properties for a specific Einstein-Maxwell-dilaton gravity theory, Phys. Rev. D 104 (2021) 026016 [arXiv: 2007.06001] [INSPIRE].

[35] Y.-Z. Li, C.-Y. Zhang and X.-M. Kuang, Entanglement wedge cross section with Gauss-Bonnet corrections and thermal quench, arXiv:2102.12171 [INSPIRE].

[36] H. Gong, P. Liu, G. Fu, X.-M. Kuang and J.-P. Wu, Informational properties of holographic Lifshitz field theory, Chin. Phys. C 45 (2021) 065101 [arXiv: 2009.00450] [InSPIRE].

[37] P. Liu, C. Niu and J.-P. Wu, The effect of anisotropy on holographic entanglement entropy and mutual information, Phys. Lett. B 796 (2019) 155 [arXiv: 1905.06808] [INSPIRE].

[38] P. Liu, Y. Ling, C. Niu and J.-P. Wu, Entanglement of purification in holographic systems, JHEP 09 (2019) 071 [arXiv: 1902. 02243] [INSPIRE].

[39] A. Lala, Entanglement measures for nonconformal D-branes, Phys. Rev. D 102 (2020) 126026 [arXiv: 2008.06154] [INSPIRE].

[40] N. Bao and I.F. Halpern, Conditional and multipartite entanglements of purification and holography, Phys. Rev. D 99 (2019) 046010 [arXiv:1805.00476] [INSPIRE].

[41] K. Umemoto and Y. Zhou, Entanglement of purification for multipartite states and its holographic dual, JHEP 10 (2018) 152 [arXiv: 1805. 02625] [INSPIRE].

[42] K. Umemoto, Quantum and classical correlations inside the entanglement wedge, Phys. Rev. D 100 (2019) 126021 [arXiv: 1907.12555] [InSPIRE]. 
[43] C. Akers and P. Rath, Entanglement wedge cross sections require tripartite entanglement, JHEP 04 (2020) 208 [arXiv:1911.07852] [INSPIRE].

[44] P. Jain and S. Mahapatra, Mixed state entanglement measures as probe for confinement, Phys. Rev. D 102 (2020) 126022 [arXiv:2010.07702] [InSPIRE].

[45] C.A. Agón, J. De Boer and J.F. Pedraza, Geometric aspects of holographic bit threads, JHEP 05 (2019) 075 [arXiv: 1811.08879] [inSPIRE].

[46] R. Espíndola, A. Guijosa and J.F. Pedraza, Entanglement wedge reconstruction and entanglement of purification, Eur. Phys. J. C 78 (2018) 646 [arXiv:1804.05855] [InSPIRE].

[47] J. Kumar Basak, V. Malvimat, H. Parihar, B. Paul and G. Sengupta, On minimal entanglement wedge cross section for holographic entanglement negativity, arXiv: 2002 .10272 [INSPIRE].

[48] J. Kumar Basak, H. Parihar, B. Paul and G. Sengupta, Covariant holographic negativity from the entanglement wedge in $A d S_{3} / C F T_{2}$, arXiv:2102.05676 [INSPIRE].

[49] K. Babaei Velni, M.R. Mohammadi Mozaffar and M.H. Vahidinia, Some aspects of entanglement wedge cross-section, JHEP 05 (2019) 200 [arXiv: 1903.08490] [INSPIRE].

[50] A. Saha and S. Gangopadhyay, Holographic study of entanglement and complexity for mixed states, Phys. Rev. D 103 (2021) 086002 [arXiv:2101.00887] [INSPIRE].

[51] K. Babaei Velni, M.R. Mohammadi Mozaffar and M.H. Vahidinia, Evolution of entanglement wedge cross section following a global quench, JHEP 08 (2020) 129 [arXiv:2005.05673] [INSPIRE].

[52] S.H. Shenker and D. Stanford, Black holes and the butterfly effect, JHEP 03 (2014) 067 [arXiv: 1306.0622] [INSPIRE].

[53] Y. Sekino and L. Susskind, Fast scramblers, JHEP 10 (2008) 065 [arXiv:0808.2096] [INSPIRE].

[54] J. Maldacena, S.H. Shenker and D. Stanford, A bound on chaos, JHEP 08 (2016) 106 [arXiv: 1503.01409] [INSPIRE].

[55] A. Donos and S.A. Hartnoll, Interaction-driven localization in holography, Nature Phys. 9 (2013) 649 [arXiv: 1212.2998] [INSPIRE].

[56] M. Blake, Universal charge diffusion and the butterfly effect in holographic theories, Phys. Rev. Lett. 117 (2016) 091601 [arXiv: 1603.08510] [INSPIRE].

[57] M. Blake, Universal diffusion in incoherent black holes, Phys. Rev. D 94 (2016) 086014 [arXiv: 1604.01754] [INSPIRE].

[58] Y. Ling, P. Liu and J.-P. Wu, Holographic butterfly effect at quantum critical points, JHEP 10 (2017) 025 [arXiv: 1610.02669] [INSPIRE].

[59] Y. Ling, P. Liu and J.-P. Wu, Note on the butterfly effect in holographic superconductor models, Phys. Lett. B 768 (2017) 288 [arXiv:1610.07146] [InSPIRE].

[60] S.-F. Wu, B. Wang, X.-H. Ge and Y. Tian, Collective diffusion and quantum chaos in holography, Phys. Rev. D 97 (2018) 106018 [arXiv:1702.08803] [INSPIRE].

[61] D. Vegh, Holography without translational symmetry, arXiv:1301.0537 [INSPIRE].

[62] M. Blake and D. Tong, Universal resistivity from holographic massive gravity, Phys. Rev. D 88 (2013) 106004 [arXiv: 1308.4970] [InSPIRE]. 
[63] M. Blake, D. Tong and D. Vegh, Holographic lattices give the graviton an effective mass, Phys. Rev. Lett. 112 (2014) 071602 [arXiv:1310.3832] [INSPIRE].

[64] R.A. Davison, Momentum relaxation in holographic massive gravity, Phys. Rev. D 88 (2013) 086003 [arXiv: 1306.5792] [INSPIRE].

[65] C. de Rham, Massive gravity, Living Rev. Rel. 17 (2014) 7 [arXiv:1401.4173] [INSPIRE].

[66] M. Baggioli and O. Pujolàs, Electron-phonon interactions, metal-insulator transitions, and holographic massive gravity, Phys. Rev. Lett. 114 (2015) 251602 [arXiv:1411.1003] [INSPIRE].

[67] L. Alberte, M. Baggioli, A. Khmelnitsky and O. Pujolàs, Solid holography and massive gravity, JHEP 02 (2016) 114 [arXiv:1510.09089] [INSPIRE].

[68] X.-X. Zeng, H. Zhang and L.-F. Li, Phase transition of holographic entanglement entropy in massive gravity, Phys. Lett. B 756 (2016) 170 [arXiv:1511.00383] [InSPIRE].

[69] R.-G. Cai, Y.-P. Hu, Q.-Y. Pan and Y.-L. Zhang, Thermodynamics of black holes in Massive Gravity, Phys. Rev. D 91 (2015) 024032 [arXiv: 1409.2369] [InSPIRE].

[70] J. Xu, L.-M. Cao and Y.-P. Hu, P-V criticality in the extended phase space of black holes in massive gravity, Phys. Rev. D 91 (2015) 124033 [arXiv:1506.03578] [InSPIRE].

[71] P. Liu and J.-P. Wu, Mixed state entanglement and thermal phase transitions, arXiv:2009.01529 [INSPIRE].

[72] M.A. Nielsen and I. Chuang, Quantum computation and quantum information, Cambridge University Press, Cambridge U.K. (2002), p. 558.

[73] J.P. Boyd, Chebyshev and Fourier spectral methods, Dover Publication, U.S.A. (2001).

[74] R. Brito, V. Cardoso and P. Pani, Superradiance: new frontiers in black hole physics, Lect. Notes Phys. 906 (2015) pp.1 [arXiv:1501.06570] [InSPIRE].

[75] R.-G. Cai, L. Li, L.-F. Li and R.-Q. Yang, Introduction to holographic superconductor models, Sci. China Phys. Mech. Astron. 58 (2015) 060401 [arXiv:1502.00437] [INSPIRE]. 